EDITORIAL

\title{
Non-contact left ventricular endocardial mapping for cardiac resynchronisation therapy: a "slow conduction" towards the fast solution
}

\author{
P Della Bella, C Carbucicchio
}

Heart 2004;90:483-484. doi: 10.1136/hrt.2003.026393

Cardiac resynchronisation therapy can help to improve left ventricular function in patients with heart failure, but only if those regions of myocardium which are mostly compromised by electromechanical desynchronisation can be identified and effectively stimulated

See end of article for authors' affiliations

Correspondence to:

Dr Paolo Della Bella, Department of Arrhythmology and Electrophysiology, Centro Cardiologico - Fondazione Monzino, IRCCS, Institute of Cardiology, University of Milan, 20138 Milan, Italy; paolo.dellabella@ ccfm. it
C ardiac resynchronisation therapy (CRT) is a new treatment strategy directed at improving left ventricular function in patients with congestive heart failure (CHF), by means of left ventricular pacing. CRT aims to correct the left ventricular conduction delay mostly observed in these patients, which can contribute to the interventricular and intraventricular mechanical asynchrony, and may therefore represent a primary curative approach. ${ }^{12}$

Important multicentred studies have already established the effectiveness of CRT as treatment for CHF, by enhancing left ventricular function parameters and by improving quality of life and exercise tolerance. ${ }^{34}$ However, there is concern over the relatively small number of patients so far enrolled in controlled trials, and the presence of a significant rate of "non-responders". Reasons for failure can be related to the inability of identifying and effectively stimulating those regions of myocardium which are mostly compromised by electromechanical desynchronisation. $^{5}{ }^{6}$

In fact, the lead applied for CRT is empirically placed in different positions over a relatively wide area of the lateral and posterolateral epicardial wall of the left ventricle, guided by the variable anatomy of the coronary veins. ${ }^{78}$

\section{IDENTIFYING THE OPTIMAL STIMULATION SITE}

The use of the surface ECG is of limited value to guide the placement of the coronary sinus lead because it only measures the time of activation," and it has been shown that similar left bundle branch block (LBBB) patterns are related to different conditions of activation of the left ventricle. ${ }^{10}{ }^{11}$ Haemodynamic parameters proved to be useful to define optimal pacing intervals ${ }^{12}$ and to evaluate the acute response from different sites of pacing, ${ }^{5}$ while new echocardiographic techniques showed additional importance in order to characterise candidates for $\mathrm{CRT}^{13-16}$; however, no method has so far been established to identify the optimal stimulation site in relation to the intraventricular defect of conduction and to long term efficacy.

Activation mapping has been only occasionally used to characterise the electrical pattern underlying the intraventricular conduction disturbance in each patient, and to identify those areas of delayed activation that benefit from local pacing. ${ }^{17}$ In this setting, a dynamic mapping displaying the sequence of depolarisation could theoretically be of great value in identifying lines of block, in measuring activation intervals in different areas, and in estimating the effect of pacing from different sites.

The paper by Lambiase and colleagues $^{18}$ recently published in Heart shows that the use of "non-contact" mapping ${ }^{19}$ adds significant elements to optimise CRT: the system allows the three dimensional reconstruction of the whole depolarisation process that takes part in the failing ventricle, and characterises areas of scar tissue and delayed activation, thereby defining the substrate of LBBB. This might represent the first step toward supporting a rational choice of a pacing site to restore an effective pattern of activation. The authors showed that areas of abnormal electrical activation are characterised by low amplitude electrograms and by prolonged propagation of depolarisation; furthermore, they proved that a significant haemodynamic improvement can be achieved by CRT only when left ventricular stimulation is applied outside of these "slow conduction" areas. When pacing is provided from within these areas, the recruitment of an adequate amount of tissue is compromised by the prolonged conduction throughout the paced area, making CRT less effective. In order to limit the harmful effect of delayed conduction from areas of slow conduction, Lambiase and colleagues $^{18}$ showed that in these patients the left chamber must be pre-excited by stimulating the left ventricle $32 \mathrm{~ms}$ before the right ventricle, causing the best coupling for the two ventricles; results of "non-contact" mapping will be compared in the future with those achieved by other techniques suggesting individually tailored preactivation of myocardium. ${ }^{16}$

\section{"NON-CONTACT" MAPPING}

Areas characterised by pathological electrograms detected by "non-contact" mapping identify a

Abbreviations: CHF, congestive heart failure; CRT, cardiac resynchronisation therapy; LBBB, left bundle branch block 
line of "fixed" conduction block: this may justify why areas of "slow conduction" are peculiar to patients with ischaemic cardiomyopathy who have suffered from previous myocardial damage, in whom effective pacing is limited by multiple anatomical barriers. In this setting, "non-contact" mapping should more accurately characterise the nature and the extension of conduction block, and act as a guide to the most effective place of pacing, both from within the coronary veins or from a "true" epicardial site. Because of the limited number of cases, however, Lambiase and colleagues ${ }^{18}$ were not able to prove a direct correlation between areas of previous infarct and the site of "block" in their patient population: alternatively, one may suppose that a more diffuse endocardial damage of the specialised conduction system may be represented in patients with ischaemic cardiomyopathy. ${ }^{10}$ Patients with idiopathic dilated cardiomyopathy, on the other hand, would present with a more functional disorder, that can be more easily corrected by means of local pacing. Further work is needed to clarify this issue.

Based on the experience of Lambiase and colleagues, ${ }^{18}$ it is reasonable to expect that "non-contact" mapping in patients with LBBB who are candidates for CRT might be used to screen patients who benefit from this treatment. A precise, more "qualitative" characterisation of the pattern of activation, also considering the analysis of the electromechanical efficiency for different segments of the left chamber, will further shed light on the most suitable site for stimulation and clarify the importance of different future strategies for pacing, including multisite stimulation. ${ }^{20}$

The major contribution offered by Lambiase and colleagues $^{18}$ is that a "pure" electrophysiological approach can guide CRT, given the extraordinary opportunity to look directly into the electrical pattern of left ventricular activation; left ventricular activation intervals can be reliably used to define the effectiveness of resynchronisation, and to guide lead implant for CRT, with a "slow" conduction being the key for future solutions.

\section{Authors' affiliations}

P Della Bella, C Carbucicchio, Department of Arrhythmology and Electrophysiology, Centro Cardiologico - Fondazione Monzino, IRCCS, Institute of Cardiology, University of Milan, Milan, Italy

\section{REFERENCES}

1 Nelson GS, Berger RD, Fetics BJ, et al. Left ventricular or biventricular pacing improves cardiac function at diminished energy cost in patients with dilated cardiomyopathy and left bundle-branch block. Circulation 2000; 102:3053-9.

2 Yu CM, Chau E, Sanderson JE, et al. Tissue Doppler echocardiographic evidence of reverse remodeling and improved synchronicity by simultaneously delaying regional contraction after biventricular pacing therapy in heart failure. Circulation 2002; 105:438-45.

3 Cazeau S, Leclercq C, Lavergne T, et al. for the MUSTIC Study Investigators. Effect of multisite biventricular pacing in patients with heart failure and intraventricular conduction delay. N Engl J Med 2001;344:873-80.

4 Abraham WT, Fisher WG, Smith AL, et al. for the MIRACLE Study Group. Cardiac resynchronisation in chronic heart failure. N Engl J Med 2002;346:1845-53.

5 Butter C, Auricchio A, Stellbrink C, et al. Effect of resynchronization therapy stimulation site on the systolic function of heart failure patients. Circulation 2001;104:3026-9.

6 Breithardt OA, Stellibrink C, Franke A, et al. Echocardiographic evidence of hemodynamic and clinical improvement in patients paced for heart failure. Am J Cardiol 2000;86:133K-7K.

7 Auricchio A, Klein H, Tockman B, et al. Transvenous biventricular pacing for heart failure: can the obstacles be overcome? Am J Cardiol 1999;83:136D-42D.

8 Meisel E, Pfeiffer D, Engelmann L, et al. Investigation of coronary venous anatomy by retrograde venography in patients with malignant ventricular tachycardia. Circulation 2001; 104:442-7.

9 Leclercq C, Faris O, Tunin R, et al. Systolic improvement and mechanical resynchronization does not require electrical synchrony in the dilated failing heart with left bundle-branch block. Circulation 2002;106:1760-3.

10 Vassallo JA, Cassidy DM, Marchlinski FE, et al. Endocardial activation of left bundle branch block. Circulation 1984;69:914-23.

11 Kass DA, Chen CH, Curry C, et al. Improved left ventricular mechanics from acute VDD pacing in patients with dilated cardiomyopathy and ventricular conduction delay. Circulation 1999;99:1567-73.

12 Auricchio A, Stellbrink C, Block M, et al. Effect of pacing chamber and atrioventricular delay on acute systolic function of paced patients with congestive heart failure. Circulation 1999;99:2993-3001.

13 Sogaard P, Egeblad H, Kim WY, et al. Tissue Doppler imaging predicts improved systolic performance and reversed left ventricular remodeling during long-term cardiac resynchronization therapy. J Am Coll Cardiol 2002;40:723-30.

14 Bax JJ, Molhoek SG, Marwick TH, et al. Usefulness of myocardial tissue Doppler echocardiography to evaluate left ventricular dyssynchrony before and after biventricular pacing in patients with idiopathic dilated cardiomyopathy. Am J Cardiol 2003;91:94-7.

15 Breithardt OA, Stellbrink C, Franke A, et al. Acute effects of cardiac resynchronization therapy on left ventricular Doppler indices in patients with congestive heart failure. Am Heart J 2002;143:34-44.

16 Sogaard P, Egeblad H, Pedersen AK, et al. Sequential versus simultaneous biventricular resynchronization for severe heart failure. Evaluation by tissue Doppler imaging. Circulation 2002;106:2078-84.

17 Auricchio A, Geller CJ, Kloss M, et al. Cardiac resynchronization therapy guided by 3-D electroanatomic nonfluoroscopic mapping (abstract). Pacing Clin Electrophysiol 2001;24:736

18 Lambiase PD, Rinaldi A, Hauck J, et al. Non-contact left ventricular endocardial mapping in cardiac resynchronization therapy. Heart 2004;90:44-51.

19 Schilling RJ, Peters NS, Davies DW. Simultaneous endocardial mapping in the human left ventricle using a noncontact catheter. Comparison of contact and reconstructed electrograms during sinus rhythm. Circulation 1998;98:887-98.

20 Pappone C, Rosanio S, Oreto G, et al. Cardiac pacing in heart failure patients with left bundle branch block: impact of pacing site for optimising left ventricular resynchronization. Ital Heart J 2000;1(7):464-9. 